\title{
Optimization of Lipase Production from Bacillus thuringiensis (TS11BP), Achromobacter xylosoxidans J2 (TS2MCN)-Isolated from Soil sediments near oilseed farm
}

\author{
Mohammad Badrud Duza ${ }^{\# 1}$, Dr. S Mastan ${ }^{2}$ \\ ${ }^{1}$ Research Scholar, Dept. of Biotechnology, Acharya Nagarjuna University, Guntur - 522510 \\ ${ }^{2}$ Post-Graduate Department of Biotechnology, PG Courses and Research Centre, DNR College, Bhimavaram-
} 534202. West Godavari Dist., A.P.

\begin{abstract}
:
Aim: The aim of the present study is to estimate the optimum growth conditions of bacterial isolates TS11BP and TS2MCN having lipolytic activity.

Materials and methods: Oil degrading bacteria were isolated from soil sample using serial dilution and pour plate method. Bacteria were further identified by morphological and biochemical tests and subjected to lipase production in $250 \mathrm{ml}$ Erlenmeyer flask using Rhodamine olive oil medium and Tween 80 medium for $48 \mathrm{~h}$ of fermentation period at $37^{0} \mathrm{C}$ with agitation speed of $150 \mathrm{rpm}$ in rotator shaker.

Results: Different bacterial strains were isolated and screened for lipase production in submerged fermentation process. Among these two bacterial strains gave positive test for lipase production. The two strains were further characterized by biochemical and morphological tests and identified as Bacillus thuringiensis (TS11BP) and Achromobacter xylosoxidans (TS2MCN). Supplementation of various bacterial growth components such as Carbon, Nitrogen, Vitamin and Amino acids increased enzyme production. The optimum $\mathrm{pH}$ and temperature for the lipase activity of crude enzyme was 8 and $45^{\circ} \mathrm{C}$ for both the bacterial isolates. Maximum lipase production was recorded during $96 \mathrm{hr}$ of incubation period and $14 \%$ of inoculums level.
\end{abstract}

\section{Introduction}

Lipases (E.C.3.1.1.3) are a group of hydrolytic enzymes that catalyze the degradation of triacylglycerols to diacylglycerol, mono-acylglycerol, fatty acids and glycerol's at the interface between aqueous and the lipid phase ${ }^{[1,2]}$. They are widely spread in nature, they were isolated from different source such as plants, animals and microorganisms; Lipase have immense potential application in various industries like cosmetic, food, detergent, paper and pharmaceutical industries ${ }^{[3,4,5,6]}$.

Enantiomers of lipase are used for resolution of chiral drugs. Biofuels products and flavor enhancers ${ }^{[7]}$. Bacterial lipases are extensively used in foodindustry for quality improvement, dairy industry for hydrolysis of milk fat, cheese ripening, in beveragesto improve aroma and in health foods for transesterification. In detergent industry for removal of oil stains from fabrics by hydrolysis of fats, ${ }^{[8]}$ in textile industry to increase fabric absorbency ${ }^{[9]}$.

In addition the enzyme is used in chemical industry for enantio selectivity and synthesis of different products. In pharmaceuticals monoglycerides are used as binders in tablets enabling slow drug release, for the synthesisof biodegradable polymers or compounds ${ }^{[10]}$ in pulp and paper industry to improve quality ${ }^{[1]]}$ degreasingleather in leather industry and for the production of various products in cosmetic industry ${ }^{[12]}$. Microbial lipases have received much more attention in industry mainly because of the availability of a wide range of hydrolytic and synthetic activities, the high yields possible, ease of genetic manipulation, regular supply due to absence of seasonal fluctuations and easy cultivation of microbes on inexpensive media. ${ }^{[13}$, ${ }^{14}$ Lipases of fungal and bacterial origin are widely used in industrial applications. Some important lipase producing bacterial genera include Bacillus, Pseudomonas and Burkholderiaetc. Lipase/esterase-producing bacteria have been found in diverse habitats such as soil contaminated with oil, dairy waste, industrial wastes, oil seeds and decaying food, compost heaps, coal tips and hot springs ${ }^{\left[15,{ }^{16]}\right.}$.The demand for the biocatalysts with novel and specific properties such as specificity, stability, $\mathrm{pH}$, and temperature is increasing day by day ${ }^{[17}$, ${ }^{18]}$. Currently bacterial lipases are of great demand because of potential industrial applications. The present paper focused on screening and isolation of microorganisms and optimization of different growth parameters for maximal lipase activity. 


\subsection{Sample collection}

\section{Materials And Methods}

Bacteria were isolated from soil collected from different sources such as soil near to oil refineries, some samples are collected from soil exposed to different oils i.e. rice bran oil, vegetable oil,palm oil,engine oil for long periods, some samples were collected from Jatropa based bio-diesel processing plant, soil samples were also collected from garbage sites comprising waste from kitchen, restaurant,market and diaries.

\subsection{Isolation of lipase producing bacteria}

Soil samples were serially diluted with sterile distilled water and spread on the nutrient agar plates followed by incubation for $24-48 \mathrm{~h}$ at $37^{\circ} \mathrm{C}$ for the growth of microorganisms. Microbial colonies, which appeared on nutrient agar plates, were purified and subjected to qualitative screening for identification of lipase/esterase producing microorganisms on Rhodamine olive oil (ROA) and tween 80 agar plates.

\subsection{Identification of lipase producing bacteria}

The bacteria were characterized morphologically based on Bergey's manual of systemic bacteriology ${ }^{[19]}$ and $16 \mathrm{~S}$ rDNA analysis.

\subsubsection{Biochemical characterization of bacterial isolates}

Different biochemical tests have been performed on bacterial isolates for classifying them according to their biochemical features. The tests include Citrate test, Methyl red test, Urease activity test, Indole test, Oxidase test, Catalase test, Vogue-Proskauer test, Gelatin test, Casein hydrolysis test, Congo red test, Motility test, Amylase test, Nitrate reduction test, Carbohydrate fermentation test by standard methods ${ }^{[20]}$.

\subsubsection{S rRNA sequencing and data analysis:}

Sequencing of RNA was performed by 1500 bp PCR product. For the sequence analysis ABI automated sequencer was used. The two 16S rRNA sequences were aligned and compared with other 16S rRNA genes in the GenBank by using the NCBI Basic Local Alignment Search Tools (BLAST).

\subsection{Screening of lipase producing Bacteria}

A sensitive and specific plate assay for detection of lipase producing bacteria makes use of rhodamineolive oil-agar medium. The growth medium contained $(\mathrm{g} / \mathrm{L})$ : nutrient broth, $8.0 ; \mathrm{NaCl}, 4.0$ and agar-agar 20 . The medium was adjusted to $\mathrm{pH} 7.0$, autoclaved and cooled to about $60^{\circ} \mathrm{C}$. Then, $31.25 \mathrm{ml}$ of olive oil and 10 $\mathrm{ml}$ of rhodamine B solution $(1.0 \mathrm{mg} / \mathrm{ml}$ distilled water and sterilized by filtration) was added with vigorous stirring. It was then pouredinto petri plates under aseptic conditions and allowed to solidify. The bacterial culture was inoculated on to themedium in these plates. Lipase producing strains were identified on spread plates after incubation for $48 \mathrm{~h}$ at $37^{\circ} \mathrm{C}$. The hydrolysis of substrate causes the formation of orange fluorescent halos around bacterial colonies visible upon UV irradiation. Secondary screening of lipolytic bacterial strains were carried out using Tween 80 hydrolysis,medium composed of (g/l): peptone, $10 ; \mathrm{NaCl}, 5 ; \mathrm{CaCl}_{2} .2 \mathrm{H}_{2} \mathrm{O}, 0.1$; agar-agar, 20; tween $80,10 \mathrm{ml}(\mathrm{v} / \mathrm{v})$.

\subsection{Lipase activity assay}

Lipase activity was assayed quantitatively by using para-nitro phenyl palmitate (pNPP) as the substrate $^{[21]}$. Tenmilliliter of isopropanol containing $30 \mathrm{mg}$ pNPP (Sigma) was mixed with $90 \mathrm{ml} 0.05 \mathrm{M}$ sodium phosphate buffer $(\mathrm{pH} 8)$ containing $207 \mathrm{mg}$ sodium deoxycholate and $100 \mathrm{mg}$ gum arabic. A total volume of 2.4 $\mathrm{ml}$ freshly preparedsubstrate solution was prewarmed at $37^{\circ} \mathrm{C}$ and mixed with $0.1 \mathrm{ml}$ enzyme solution. After 15 min incubation at $37^{\circ} \mathrm{C}$, absorbance at $410 \mathrm{~nm}$ was measured against the blank. One enzyme unit was defined as amount of enzyme requiredto release $1 \mu \mathrm{mol}$ of $\mathrm{p}$-nitrophenol from the substrate in milliliters per minute.

\subsection{Optimization of growth parameters for lipase production}

Eight independent parameters carbon sources, nitrogen sources, $\mathrm{pH}$, temperature, Incubation time, inoculum level, vitamins and amino acids supplement have significant influence on Lipase activity.

Different carbon sources such as Glucose, Fructose, Sucrose, Xylose, Mannitol, Mannose, Arabinose and Dextrose were added to the basal medium in $0.50 \%$ and $1.0 \%$ concentration. Different Nitrogen sources such as peptone, yeast extract, tryptone, Soyabean Meal, Beef extract and Gelatin were added to the basal medium in $0.50 \%$ and $1.0 \%$ concentration.

For optimizing $\mathrm{pH}$, temperature, incubation time and inoculums level different values of $\mathrm{pH}$ ranging from 3 to 11 has been maintained and tested, temperature optimization studies are carried out in $20^{\circ} \mathrm{C}$ to $60^{\circ} \mathrm{C}$ range, incubation time was maintained in the range of $24,48,72,96,120,144$ and 168 , in order to optimize 
inoculums level different $\%$ of inoculums was added to the media.Not only the conventional parameters $\mathrm{pH}$, temperature and above mentioned growth parameters but also micro nutrients are also optimized for lipase production. In this regard Vitamins such as Riboflavin, Ascorbic acid, Biotin, PyrodoxineHCl, Folic acid, Nicotinic acid and ThamineHCl, Amino acids such as L-Lysine, L-Luecine, L-Histidine, Tyrosine, Tryptophan, Aspargine, Glycine, D-Alanine, Cysteine, Arginine and Phenylalanine were added in $0.01 \%$ concentration to the media.

\section{Results And Discussions}

3.1 Isolation of Lipase producing bacteria:

Two bacteria out of 17 isolates (TS2MCN and TS11BP) from different soil samples were screened as Lipase producing bacteria and showing clear zone on Rhodamine agar plate.

Table 1:Screening of different enzymes producing bacterial strains

\begin{tabular}{|c|c|c|c|c|c|}
\hline \multirow[t]{2}{*}{ S.No } & \multirow[t]{2}{*}{ Organism tested } & \multicolumn{4}{|c|}{ Result } \\
\hline & & Amylase & Protease & Cellulase & Lipase \\
\hline 1 & TS1MCP & $-\mathrm{ve}$ & $+\mathrm{ve}$ & $-\mathrm{ve}$ & -ve \\
\hline 2 & TS2MCN & -ve & -ve & -ve & +ve \\
\hline 3 & TS3BP & $+\mathrm{ve}$ & $+\mathrm{ve}$ & -ve & -ve \\
\hline 4 & TS4BP & -ve & $+\mathrm{ve}$ & -ve & -ve \\
\hline 5 & TS5SRP & $+\mathrm{ve}$ & -ve & $+\mathrm{ve}$ & -ve \\
\hline 6 & TS6MCN & +ve & -ve & -ve & -ve \\
\hline 7 & TS7BN & -ve & $+\mathrm{ve}$ & -ve & -ve \\
\hline 8 & TS8MCN & -ve & $+\mathrm{ve}$ & -ve & -ve \\
\hline 9 & TS9MCN & -ve & $+\mathrm{ve}$ & -ve & -ve \\
\hline 10 & TS10MCN & $+\mathrm{ve}$ & -ve & -ve & -ve \\
\hline 11 & TS11BP & -ve & -ve & -ve & +ve \\
\hline 12 & TS12BN & -ve & $+\mathrm{ve}$ & -ve & -ve \\
\hline 13 & TS13SP & +ve & -ve & -ve & -ve \\
\hline 14 & TS14SN & $+\mathrm{ve}$ & $+\mathrm{ve}$ & -ve & -ve \\
\hline 15 & TS15MCN & $+\mathrm{ve}$ & -ve & -ve & -ve \\
\hline 16 & TS16MCN & + ve & -ve & $+\mathrm{ve}$ & -ve \\
\hline 17 & TS17BP & $+\mathrm{ve}$ & -ve & -ve & -ve \\
\hline
\end{tabular}

\subsection{Identification of Isolated bacterial strains:}

These bacterial isolates were characterized on the basis of colony characteristics, microscopic appearance and biochemical tests. Molecular characterization of these strains was done by 16S rRNA analysis. Further these amplified 16S rRNA sequences of the bacterial strains was blasted using online tool (MEGA 4). The taxonomical identification was done by the phylogenetic tree construction and the comparison of these bacterial strain sequences with other homologous bacterial sequences. After morphological, physiological, biochemical and taxonomical identification, these Two bacterial isolates were identified as Achromobacter xylosoxidans(TS2MCN) and Bacillus thuringiensis (TS11BP). 
Optimization of Lipase Production from Bacillus thuringiensis (TS11BP), Achromobacter

Table 2: Morphological and biochemical characters of strains isolated from soil sample

\begin{tabular}{|c|c|c|c|c|c|c|c|c|c|c|c|c|c|c|c|c|c|c|}
\hline $\begin{array}{l}\mathbf{S} . \\
\mathbf{N} \\
\mathbf{0}\end{array}$ & $\begin{array}{l}\text { Strain Code } \rightarrow \\
\text { Name of the Test }\end{array}$ & $\begin{array}{l}\text { T } \\
\text { S1 } \\
\text { M } \\
\text { C } \\
\mathbf{P}\end{array}$ & $\begin{array}{c}\mathbf{T} \\
\mathbf{S} 2 \\
\mathbf{M} \\
\mathrm{C} \\
\mathbf{N}\end{array}$ & $\begin{array}{l}\mathbf{T} \\
\mathbf{S} \\
\mathbf{3} \\
\mathbf{B} \\
\mathbf{P}\end{array}$ & $\begin{array}{l}\text { T } \\
\text { S } \\
4 \\
\text { B } \\
\text { P }\end{array}$ & $\begin{array}{c}\text { T } \\
\text { S5 } \\
\text { S } \\
\text { R } \\
\text { P }\end{array}$ & $\begin{array}{c}\mathrm{T} \\
\text { S6 } \\
\mathrm{M} \\
\mathrm{C} \\
\mathrm{N}\end{array}$ & $\begin{array}{l}\text { T } \\
\text { S } \\
7 \\
\text { B } \\
\mathbf{N}\end{array}$ & $\begin{array}{c}\mathbf{T} \\
\mathbf{S} \\
\mathbf{8} \\
\mathbf{M} \\
\mathbf{C} \\
\mathbf{N}\end{array}$ & $\begin{array}{c}\mathbf{T} \\
\mathbf{S} \\
\mathbf{9} \\
\mathbf{M} \\
\mathrm{C} \\
\mathbf{N}\end{array}$ & $\begin{array}{c}\text { T } \\
\text { S1 } \\
\mathbf{0} \\
\mathbf{M} \\
\mathrm{C} \\
\mathbf{N}\end{array}$ & $\begin{array}{c}\text { T } \\
\text { S1 } \\
\text { 1B } \\
\text { P }\end{array}$ & $\begin{array}{c}\text { TS } \\
12 \\
\text { BN }\end{array}$ & $\begin{array}{c}\mathbf{T} \\
\mathbf{S} 1 \\
\mathbf{3} \\
\mathbf{S} \\
\mathbf{P}\end{array}$ & $\begin{array}{c}\text { TS } \\
14 \\
\text { SN }\end{array}$ & $\begin{array}{c}\mathbf{T} \\
\mathbf{S 1} \\
\mathbf{5} \\
\mathbf{M} \\
\mathbf{C} \\
\mathbf{N}\end{array}$ & $\begin{array}{c}T \\
\text { S1 } \\
6 \\
M \\
\text { C } \\
\text { N } \\
\end{array}$ & $\begin{array}{c}\mathrm{T} \\
\mathrm{S} 1 \\
7 \\
\mathrm{~B} \\
\mathrm{P}\end{array}$ \\
\hline 1 & Gram Staining & $\begin{array}{l}+ \\
(\mathrm{G} \\
\mathrm{P})\end{array}$ & - & $\begin{array}{l}+ \\
( \\
G \\
P)\end{array}$ & $\begin{array}{l}+ \\
( \\
\mathrm{G} \\
\mathrm{P} \\
)\end{array}$ & $\begin{array}{l}+ \\
(\mathrm{G} \\
\mathrm{P})\end{array}$ & - & - & - & - & - & $\begin{array}{l}+ \\
\text { (G } \\
\mathrm{P})\end{array}$ & - & + & - & $\begin{array}{c}+ \\
(\mathrm{G} \\
\mathrm{P})\end{array}$ & - & $\begin{array}{l}+ \\
\text { (G } \\
\mathrm{P})\end{array}$ \\
\hline 2 & Citrate utilization & + & + & + & + & + & + & + & + & + & + & + & + & + & + & + & + & + \\
\hline 3 & Urease & - & - & + & - & + & - & - & - & - & - & + & - & - & - & - & + & - \\
\hline 4 & $\mathrm{H}_{2} \mathrm{~S}$ test & - & - & + & - & - & - & + & - & - & - & - & - & - & - & - & + & - \\
\hline 5 & Oxidation & + & + & + & + & + & + & + & + & + & + & + & + & + & + & + & + & + \\
\hline 6 & Methyl Red & + & + & - & + & + & + & + & + & + & + & + & + & + & + & - & + & + \\
\hline 7 & Indole test & - & + & - & + & + & - & + & - & + & + & - & - & - & - & + & - & - \\
\hline 8 & $\begin{array}{l}\text { Vogues- } \\
\text { Proskaeur(VP) test: }\end{array}$ & - & - & + & - & + & - & + & - & - & - & + & + & + & - & - & - & - \\
\hline 9 & Catalase: & + & + & + & + & + & + & + & + & + & + & + & + & + & + & + & + & + \\
\hline $\begin{array}{l}1 \\
0\end{array}$ & Fermentation test & - & - & $\begin{array}{l}+ \\
\mathrm{G} \\
\mathrm{P}\end{array}$ & + & + & + & + & - & + & + & $\begin{array}{l}+ \\
\mathrm{G} \\
\mathrm{P}\end{array}$ & + & + & + & + & + & + \\
\hline $\begin{array}{l}1 \\
1\end{array}$ & Nitrate reductase & + & + & - & - & - & + & - & - & + & - & - & + & + & - & - & - & + \\
\hline
\end{tabular}

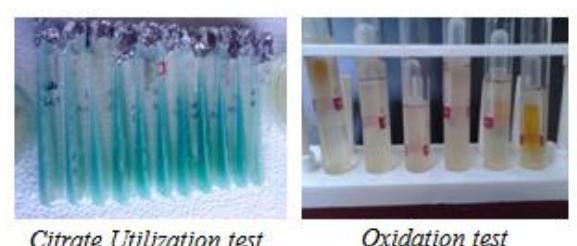

Citrate Utilization test

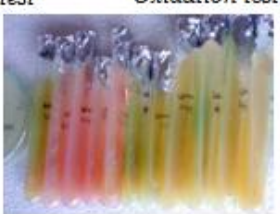

Urease Test

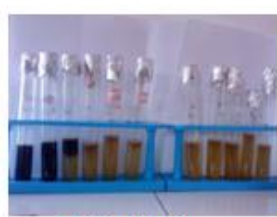

Methyl Red test

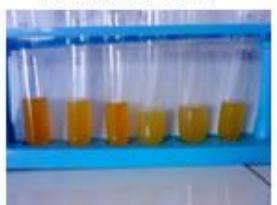

Vogues-Proskaeur(VP) test

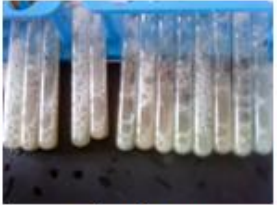

Catalase test

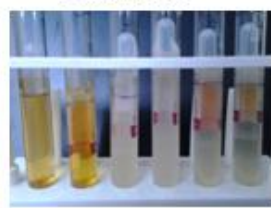

Fermentation Test

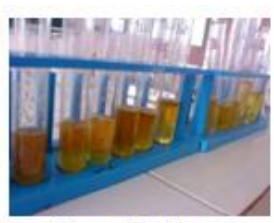

Nitrate Reductase test

Methyl red, Catalase and nitrate reductase tests confirms Monococcus strains. And Urease test, Voues-

Proskaeur (VP) test, Fermentation Tests confirms Bacillus strains.

\subsubsection{S rRNA sequencing and analysis data:}

TS2MCN is Identified as Achromobacter xylosoxidans strain using 16S ribosomal RNA gene sequence studies

GenBank: KF525350.1 GenBank Graphics PopSet

>gi|530549974|gb|KF525350.1| Achromobacter xylosoxidans TS24B 16S ribosomal RNA gene, partial

sequence

ACATGCACGTCGACCGCTGGCGGACTTCTTGCTGGTGGCAAGTGGCGGCGGGTGAGTGATGTATC

GGAAC

GTGCCCTGTCCCGGGGGAGACTTCACTCCAGCGTACCTGATACCGCACCCGCCCTACTTTGGAAAC

CCGG

GGATCTTCATACCTTGGCCTATTGGACCGGCCGATATCGAATTACCTACTTGGTGGGGTAACGGCC

TACC

AAGGCCACCATCCCTAGGTGGTTTGACAGGACCACCCTCCACGGTGGAACTGACACACTGCCCCTA

CTCC

TACGGGAGGACTCCTTGGGGAATTTTGGACAATGGGGGAAACCCTGAGCCAGACATCCCGCGTGA

GCAAC

GACGGCCTTCTGATTAAAGCTTTCTTTTGGCAAGAATCAATTGTCACGGACTAATACCTCCCAAAT

CTGA

CGGCTCCTGCCTAATCAGCACCTGATCACTACGTGCCAGCTACCTCGGTACTACCTACGGTGCAAG CGTT 
Optimization of Lipase Production from Bacillus thuringiensis (TS11BP), Achromobacter

TATCTGAATTACTGGGCGTAAAGTTTGCGCACGTGATTCGCAAGCAGGTATGTTCTATCGTATAAC GTAA

CTTTGCAACTGCCTTTCTAACTGGGGGCTAGAGAGTGTCAGACGGAGGTGCAAATCCGCGTATTGC AGTT

ACATGTGTAGATATGAAGAGGAACAACGTTTGCAAAAGCACCCTCGTGGGATGACACTGACGCTT CTGCA

CCAGAGCCTGGGGAGCAAACGCGAGTGGAGCCCCTGGGAGTCCATGCCCTGGACGATCTCAACTA ACTGG

TAGGGAGTCCTCAGTGCTTGATAGCGTACCCTCACTGTGTTGACTGTAGACCGACTGAGGAGTCAC GTCC

GCCAGATTAATACTCACAGCGAAGTCTACAGCTACCCGCACTTGCCGGGGGATCCATGCTCGATCA TGCG

ATGCGTCGCTTAAATCGTAGCTACCCGTGACTGTCTCGGAGTGCTGACTACATCTGACAATGCTCG CAA

TS11BP is identified as Bacillus thuringiensis using 16S ribosomal RNA gene, partial sequence GenBank: KF525352.1

GenBank Graphics PopSet

>gi|530549976|gb|KF525352.1| Bacillus thuringiensis strain SSME 16S ribosomal RNA gene, partial sequence TGGATTGGAGCTTGCTCTTATGAAGTTAGCGGCGGACGGGTGAGTAACACGTGGGTAACCTGCCC ATAAG ACTGGGATAACTCCGGGAAACCGGGGCTAATACCGGATAACATTTTGAACCGCATGGTTCGAAAT TGAAA GGCGGCTTCGGCTGTCACTTATGGATGGACCCGCGTCGCATTAGCTAGTTGGTGAGGTAACGGCTC ACCA AGGCAACGATGCGTAGCCGACCTGAGAGGGTGATCGGCCACACTGGGACTGAGACACGGCCCAG ACTCCT

ACGGGAGGCAGCAGTAGGGAATCTTCCGCAATGGACGAAAGTCTGACGGAGCAACGCCGCGTGA GTGATG

AAGGCTTTCGGGTCGTAAAACTCTGTTGTTAGGGAAGAACAAGTGCTAGTTGAATAAGCTGGCAC CTTGA

CGGTACCTAACCAGAAAGCCACGGCTAACTACGTGCCAGCAGCCGCGGTAATACGTAGGTGGCAA GCGTT

ATCCGGAATTATTGCGCGTAAAGCGCGCGCAGGTGGTTTCTTAAGTCTGATGTGAAAGCCCACGGC TCAA

CCGTGGAGGGTCATTGGAAACTGGGAGACTTGAGTGCAGAAGAGGAAAGTGGAATTCCATGTGTA GCGGT

GAAATGCGTAGAGATATGGAGGAACACCAGTGGCGAAGGCGACTTTCTGGTCTGTAACTGACACT GAGGC

GCGAAAGCGTGGGGAGCAAACAGGATTAGATACCCTGGTAGTCCACGCCGTAAACGATGAGTGCT AAGTG

TTAGAGGGTTTCCGCCCTTTAGTGCTGAAGTTAACGCATTAAGCACTCCGCCTGGGGAGTACGGCC GCAA

GCTGAACTCAAAGGAATTGACGGGGCCCGCACGAGCGTGAGCATGTGTTTAATTCGAAGCACGCG AGAAC

CTACGAGTCTGACATCATCTGACACCCTAGAGATAGGGCTGCTCTCGGGAGCAGAGTGACAGCTG CTGCA

TGTGTCGTCAGCTCGGTCATGACATGTAGGGTAGTCGCTACGATCGCAGCATGGATCTAGTGGCAT CCAT

TAGTTGTCACTTACGTGACTGCGTGACTACGAGACGTGGATGACGTCAATCTCATGCTATGACCTG GCTA

ACGT 


\subsection{Assay of Lipase activity}

Estimation of enzyme activity for the positive isolates has conducted. Concentration of enzyme in crude sample was calculated by reacting with the enzyme with substrate comparing the resultant O.D with standard graph. The enzyme activity was expressed in International Unit (IU). 1U/ML = Amount of enzyme which releases 1micro mole glucose under assay conditions. The results of amylase assay are shown in Table 3 .

Table 3: Determination of Lipase activity from Lipase producing Bacteria

\begin{tabular}{lllc}
\hline S.NO & Organism tested & Type of Strain & $\begin{array}{l}\text { Lipase activity in the } \\
\text { culture supernatant IU/mg }\end{array}$ \\
\hline 1 & TS2MCN & Monococcus sp. & 3.9 \\
\hline 2 & TS11BP & Bacillus $s p$. & 1.6 \\
\hline
\end{tabular}

\subsection{Optimization Studies}

\subsubsection{Effect of different Carbon Sources on lipase production}

To determine the effect of carbon sources on lipase production by Bacillus thuringenesis(TS11BP), and Achromobacter xylosoxidans(TS2MCN) carbon sources mainly carbohydrates were screened for their efficiency to support lipase production like Glucose, Fructose, Sucrose, Xylose, Mannitol, Mannose, Arabinose and Dextrose were used as the basal carbon sources in basal medium and was assayed to check for the lipase activity.

Table 4: Effect of different Carbon sources on Lipase production by Two Strains

\begin{tabular}{|c|c|c|c|c|c|c|c|c|c|}
\hline \multicolumn{2}{|c|}{ Bacterial isolate } & \multicolumn{4}{|c|}{ Achromobacter xylosoxidans (TS2MCN) } & \multicolumn{4}{|c|}{$\begin{array}{l}\text { Bacillus thuringiensis } \\
\text { (TS11BP) }\end{array}$} \\
\hline \multirow[t]{2}{*}{ S.NO } & \multirow{2}{*}{$\begin{array}{l}\text { Carbon } \\
\text { Source }\end{array}$} & \multicolumn{2}{|c|}{$0.50 \%$} & \multicolumn{2}{|c|}{$1.0 \%$} & \multicolumn{2}{|c|}{$0.50 \%$} & \multicolumn{2}{|c|}{$1.0 \%$} \\
\hline & & $\begin{array}{c}\text { IU } \\
\text { units }\end{array}$ & $\%$ Assay & $\begin{array}{c}\text { IU } \\
\text { units }\end{array}$ & $\%$ Assay & $\begin{array}{c}\text { IU } \\
\text { units }\end{array}$ & $\%$ Assay & $\begin{array}{c}\text { IU } \\
\text { units }\end{array}$ & $\%$ Assay \\
\hline 0 & Standard & 3.90 & $\ldots$ & 3.90 & 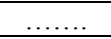 & 1.60 & & 1.60 & \\
\hline 1 & Glucose & 3.81 & 97.69 & 3.97 & 101.79 & 1.79 & 111.87 & 1.94 & 121.25 \\
\hline 2 & Fructose & 3.28 & 84.10 & 3.45 & 88.46 & 1.52 & 95.00 & 1.76 & 110.00 \\
\hline 3 & Sucrose & 1.69 & 43.33 & 1.87 & 47.94 & 0.99 & 61.87 & 1.23 & 76.87 \\
\hline 4 & Xylose & 1.52 & 38.97 & 1.72 & 44.10 & 0.79 & 49.37 & 0.86 & 53.75 \\
\hline 5 & Manitol & 2.02 & 51.79 & 2.54 & 65.12 & 1.15 & 71.87 & 1.54 & 96.25 \\
\hline 6 & Mannose & 2.55 & 65.38 & 2.76 & 70.76 & 0.76 & 47.50 & 1.10 & 68.75 \\
\hline 7 & Arabinose & 3.63 & 93.08 & 3.82 & 97.94 & 1.11 & 69.37 & 1.38 & 86.25 \\
\hline 8 & Dextrose & 4.12 & 105.641 & 4.49 & 115.12 & 1.89 & 118.12 & 2.28 & 142.50 \\
\hline
\end{tabular}

In the present study, it has been observed that both the bacterial isolates produced maximum enzyme yield in presence of Dextrose in the medium, comparatively Achromobacter xylosoxidans (TS2MCN) produced $(4.12 \mathrm{IU} / \mathrm{ml} / \mathrm{min}, 4.49 \mathrm{IU} / \mathrm{ml} / \mathrm{min}$ in $0.05 \%, 1.0 \%$ Dextrose concentrations respectively) more lipase enzyme than Bacillus thuringenesis (TS11BP) which produced $1.89 \mathrm{IU} / \mathrm{ml} / \mathrm{min}, 2.28 \mathrm{IU} / \mathrm{ml} / \mathrm{min}$ in $0.05 \%, 1.0 \%$ Dextrose concentrations, respectively.
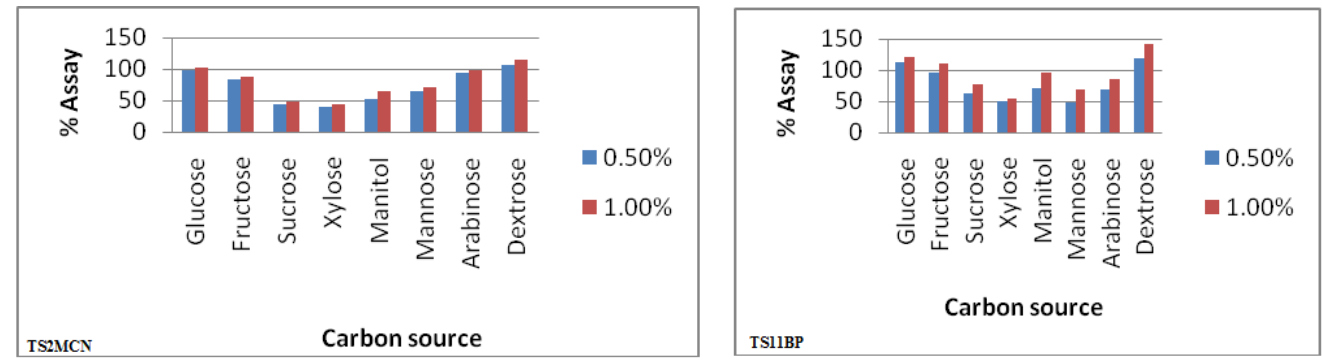

Fig.2 Effect of different carbon Sources on Lipase production by Two Strains

\subsubsection{Effect of different Nitrogen Sources on lipase production}

Optimization was carried out by using different organic nitrogen as nitrogen sources. Different nitrogen source used were peptone, yeast extract, tryptone, Soyabean Meal, Beef extract and Gelatin were added to the medium and incubated at $37^{\circ} \mathrm{C}$ for $24 \mathrm{hrs}$ in a rotary shaker. 
Table 5: Effect of different Nitrogen sources on Lipase production by Two Strains

\begin{tabular}{|c|c|c|c|c|c|c|c|c|c|}
\hline \multicolumn{2}{|r|}{ Bacterial isolate } & \multicolumn{4}{|c|}{ Achromobacter xylosoxidans (TS2MCN) } & \multicolumn{4}{|c|}{$\begin{array}{l}\text { Bacillus thuringiensis } \\
\text { (TS11BP) }\end{array}$} \\
\hline \multirow[t]{2}{*}{ S.NO } & \multirow[b]{2}{*}{ Nitrogen Source } & \multicolumn{2}{|c|}{$0.50 \%$} & \multicolumn{2}{|c|}{$1.0 \%$} & \multicolumn{2}{|c|}{$0.50 \%$} & \multicolumn{2}{|c|}{$1.0 \%$} \\
\hline & & $\begin{array}{c}\text { IU } \\
\text { units }\end{array}$ & $\%$ Assay & $\begin{array}{c}\text { IU } \\
\text { units }\end{array}$ & $\%$ Assay & $\begin{array}{c}\text { IU } \\
\text { units }\end{array}$ & $\%$ Assay & $\begin{array}{c}\text { IU } \\
\text { units }\end{array}$ & \% Assay \\
\hline 0 & Standard & 3.90 & $\ldots \ldots \ldots$ & 3.90 & $\ldots \ldots \ldots$ & 1.60 & $\ldots \ldots \ldots$ & 1.60 & $\ldots \ldots \ldots$ \\
\hline 1 & Peptone & 4.29 & 110.00 & 4.57 & 117.17 & 1.96 & 122.50 & 2.38 & 148.75 \\
\hline 2 & Yeast Extract & 3.19 & 81.79 & 3.49 & 89.48 & 1.22 & 76.25 & 1.48 & 92.50 \\
\hline 3 & Tryptone & 2.56 & 65.64 & 2.72 & 69.74 & 1.59 & 99.37 & 1.72 & 107.50 \\
\hline 4 & Soyabean Meal & 2.11 & 54.10 & 2.37 & 60.76 & 1.93 & 120.62 & 2.13 & 133.12 \\
\hline 5 & Beaf extract & 4.01 & 102.82 & 4.38 & 112.30 & 2.15 & 134.37 & 2.59 & 161.875 \\
\hline 6 & Gelatin & 3.96 & 101.54 & 4.10 & 105.12 & 1.08 & 67.50 & 1.48 & 92.50 \\
\hline
\end{tabular}

In the present study, Achromobacter xylosoxidans (TS2MCN) produced $4.29 \mathrm{U} / \mathrm{ml} / \mathrm{min}$, $4.57 \mathrm{IU} / \mathrm{ml} / \mathrm{min}$ in $0.05 \%, 1.0 \%$ peptone concentrations respectively and Bacillus thuringenesis(TS11BP) yielded $2.15 \mathrm{IU} / \mathrm{ml} / \mathrm{min}, 2.59 \mathrm{IU} / \mathrm{ml} / \mathrm{min}$ in $0.05 \%, 1.0 \%$ Beef extract concentrations. Thus for Achromobacter xylosoxidans (TS2MCN) best carbon source was Peptone whereas Beef extract was best for Bacillus thuringenesis(TS11BP) yielding maximum enzyme production.

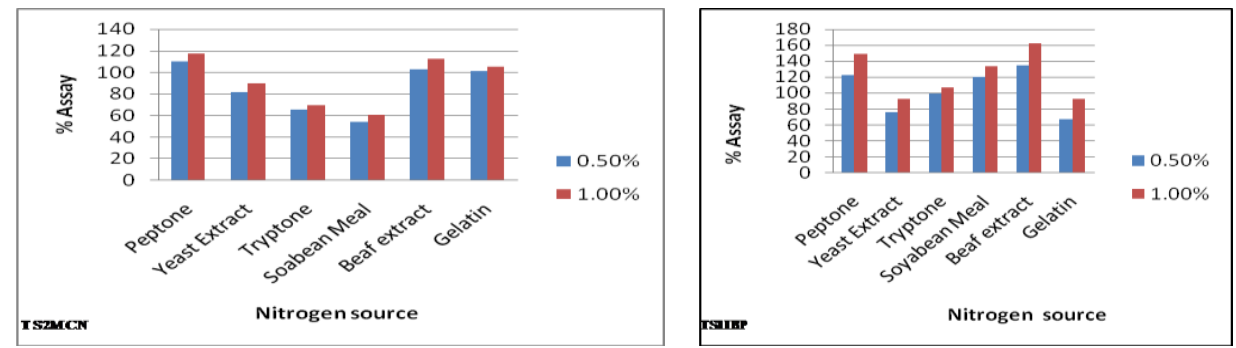

Figure 3: Effect of nitrogen sources on Lipase production by Two Strains

\subsubsection{Effect of pH and Temperature on Lipase Activity}

The optimum $\mathrm{pH}$ for enzyme production was selected by varying the $\mathrm{pH}$ of the medium from 3 to 11 . For selection of optimum temperature for the production of lipases, the temperature varying from 20 to $60^{\circ} \mathrm{C}$ were maintained and tested for optimization studies.

Table 6: Effect of pH on Lipase production by Two Strains

\begin{tabular}{|c|c|c|c|c|c|}
\hline \multicolumn{2}{|c|}{ Bacterial isolate } & \multicolumn{2}{c|}{ Achromobacter xylosoxidans (TS2MCN) } & \multicolumn{2}{c|}{$\begin{array}{c}\text { Bacillus thuringiensis } \\
\text { (TS11BP) }\end{array}$} \\
\hline S.NO & $\mathrm{pH}$ & IU units & \% Assay & IU units & $\ldots \ldots$. \\
\hline 0 & Standard & 3.90 & $\ldots \ldots \ldots$ & 1.60 & 4.75 \\
\hline 1 & 3 & 0.045 & 1.15 & 0.076 & 6.1875 \\
\hline 2 & 4 & 0.23 & 5.90 & 0.099 & 9.375 \\
\hline 3 & 5 & 0.36 & 9.23 & 0.15 & 13.75 \\
\hline 4 & 6 & 0.66 & 16.92 & 0.22 & 18.12 \\
\hline 5 & 7 & 1.26 & 32.31 & 0.29 & 117.50 \\
\hline 6 & 8 & 4.33 & 111.02 & 1.88 & 61.25 \\
\hline 7 & 9 & 2.07 & 53.08 & 0.98 & 20.62 \\
\hline 9 & 10 & 0.59 & 15.13 & 0.33 & 3.50 \\
\hline
\end{tabular}

Table 7: Effect of Temperature on Lipase production by Two Strains

\begin{tabular}{|c|c|c|c|c|c|}
\hline \multicolumn{2}{|c|}{ Bacterial isolate } & \multicolumn{2}{|c|}{ Achromobacterxylosoxidans J2 (TS2MCN) } & \multicolumn{2}{|c|}{ Bacillus thuringiensis (TS11BP) } \\
\hline S.NO & $\begin{array}{l}\text { Temperature } \\
\text { ( in }{ }^{\circ} \mathrm{C} \text { ) }\end{array}$ & IU units & $\%$ Assay & IU units & \% Assay \\
\hline 0 & Standard & 3.90 & $\ldots \ldots$ & 1.60 & $\ldots \ldots$ \\
\hline 1 & 20 & 0.17 & 4.36 & 0.11 & 6.87 \\
\hline 2 & 25 & 0.2 & 5.13 & 0.19 & 11.87 \\
\hline 3 & 30 & 0.37 & 9.49 & 0.27 & 16.87 \\
\hline 4 & 35 & 2.24 & 57.43 & 1.09 & 68.12 \\
\hline 5 & 40 & 3.56 & 91.28 & 1.28 & 80.00 \\
\hline 6 & 45 & 4.69 & 120.26 & 1.97 & 123.12 \\
\hline 7 & 50 & 2.69 & 68.97 & 1.11 & 69.37 \\
\hline 8 & 55 & 1.24 & 31.79 & 0.93 & 58.12 \\
\hline 9 & 60 & 1.11 & 28.46 & 0.05 & 3.12 \\
\hline
\end{tabular}


In the present study, maximum lipase production was obtained around $\mathrm{pH} 8$ for both of the bacterial isolates, Achromobacter xylosoxidans(TS2MCN) showed enzyme activity of $4.33 \mathrm{IU} / \mathrm{ml} / \mathrm{min}$ whereas Bacillus thuringenesis (TS11BP) produced $1.88 \mathrm{IU} / \mathrm{ml} / \mathrm{min}$. Optimum temperature for maximum lipase production was $45^{\circ} \mathrm{C}$ illustrated by enzyme assay in temperature optimization studies shown in Table 7.
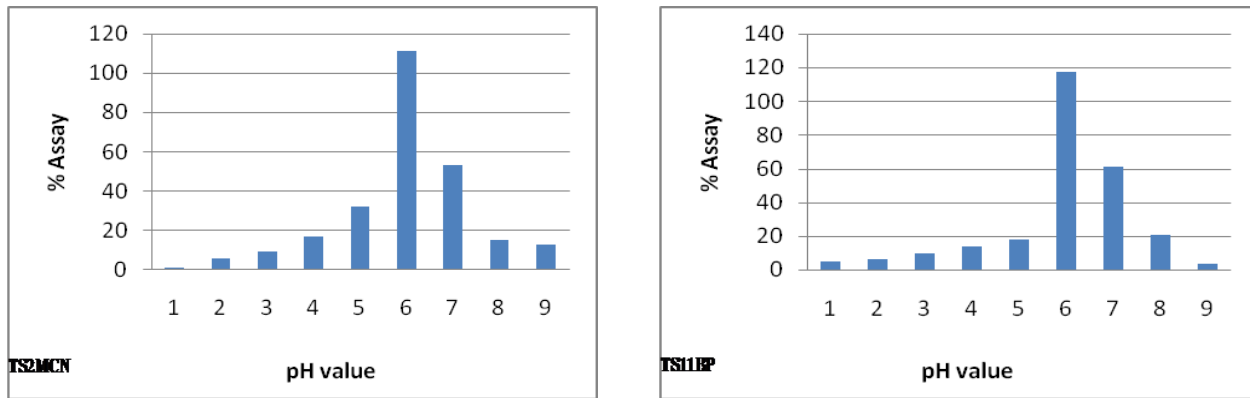

Figure 4: Effect of pH on Lipase production by Two Strains
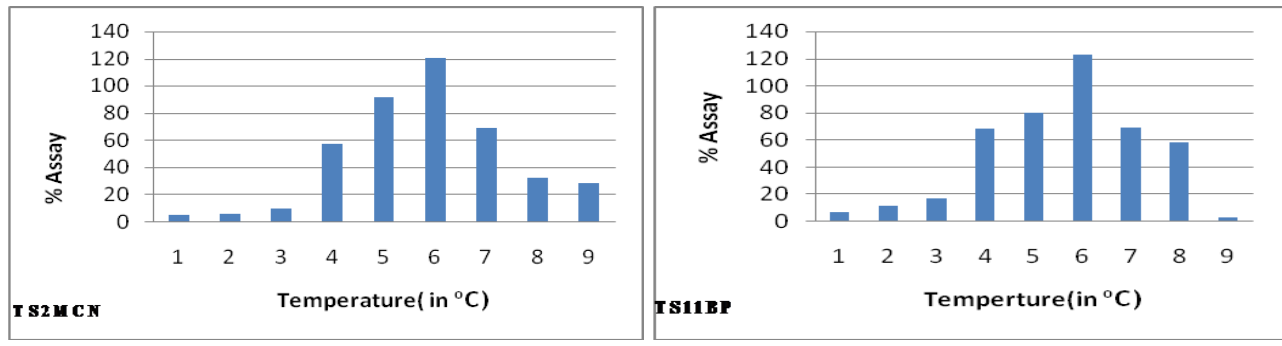

Figure 5: Effect of Temperature on Lipase production by Two Strains

\subsubsection{Effect of incubation time and inoculums level on lipase production:}

The effect of incubation time on lipase production was determined by incubating the medium for $24,48,72,96,120,144,168$ hours and the enzyme activity was assayed at different time intervals $(0-168 \mathrm{hr})$. Different inoculums size in range of $4,6,8,10,12,14$ and $16 \%$ were added to the basal medium and optimum inoculums level was tested and determined.

Table 8: Effect of Incubation time on bacterial isolates

\begin{tabular}{|c|c|c|c|c|c|}
\hline \multicolumn{2}{|c|}{ Bacterial isolate } & \multicolumn{2}{c|}{ Achromobacter xylosoxidans (TS2MCN) } & \multicolumn{2}{c|}{$\begin{array}{c}\text { Bacillus thuringiensis } \\
\text { (TS11BP) }\end{array}$} \\
\hline S.NO & $\begin{array}{c}\text { Incubation } \\
\text { time(in hr) }\end{array}$ & IU units & $\%$ Assay Assay & IU units & $\ldots \ldots$ \\
\hline 0 & Standard & 3.90 & $\ldots \ldots$ & 1.60 & 33.125 \\
\hline 1 & 24 & 1.53 & 39.23 & 0.53 & 76.25 \\
\hline 2 & 48 & 2.35 & 60.25 & 1.22 & 117.5 \\
\hline 3 & 72 & 3.89 & 99.74 & 2.88 & 127.5 \\
\hline 4 & 96 & 4.35 & 111.53 & 1.50 & 93.75 \\
\hline 5 & 120 & 2.22 & 56.92 & 1.03 & 64.37 \\
\hline 6 & 144 & 1.03 & 26.41 & 0.22 & 13.75 \\
\hline 7 & 168 & 0.55 & 14.10 & & \\
\hline
\end{tabular}

Table 9: Effect of Inoculums level on bacterial isolates

\begin{tabular}{|c|c|c|c|c|c|}
\hline \multicolumn{2}{|c|}{ Bacterial isolate } & \multicolumn{2}{c|}{ Achromobacter xylosoxidans (TS2MCN) } & \multicolumn{2}{c|}{$\begin{array}{c}\text { Bacillus thuringiensis } \\
\text { (TS11BP) }\end{array}$} \\
\hline S.NO & $\begin{array}{c}\text { Inoculums } \\
\text { level }\end{array}$ & IU units & $\%$ Assay & IU units & $\ldots \ldots$ \\
\hline 0 & Standard & 3.90 & $\ldots \ldots$ & 1.60 & 27.50 \\
\hline 1 & 4 & 1.22 & 31.28 & 0.44 & 53.75 \\
\hline 2 & 6 & 2.55 & 65.38 & 0.86 & 76.25 \\
\hline 3 & 8 & 3.22 & 82.56 & 1.22 & 104.37 \\
\hline 4 & 10 & 4.23 & 108.46 & 1.67 & 124.37 \\
\hline 5 & 12 & 4.79 & 122.82 & 1.99 & 117.50 \\
\hline 6 & 14 & 3.99 & 102.30 & 1.88 & 96.87 \\
\hline 7 & 16 & 3.55 & 91.10 & 1.55 & \\
\hline
\end{tabular}

In the present study, maximum lipase production was recorded during incubation period of $96 \mathrm{hr}$ by the Achromobacter xylosoxidans (TS2MCN) and Bacillus thuringenesis(TS11BP) as $4.35 \mathrm{IU} / \mathrm{ml} / \mathrm{min}$ and 2.04 
$\mathrm{IU} / \mathrm{ml} / \mathrm{min}$. In case of inoculums level $12 \%$ is considered as best for maximum growth of both the bacterial isolates and facilitate maximum production of lipase enzyme.

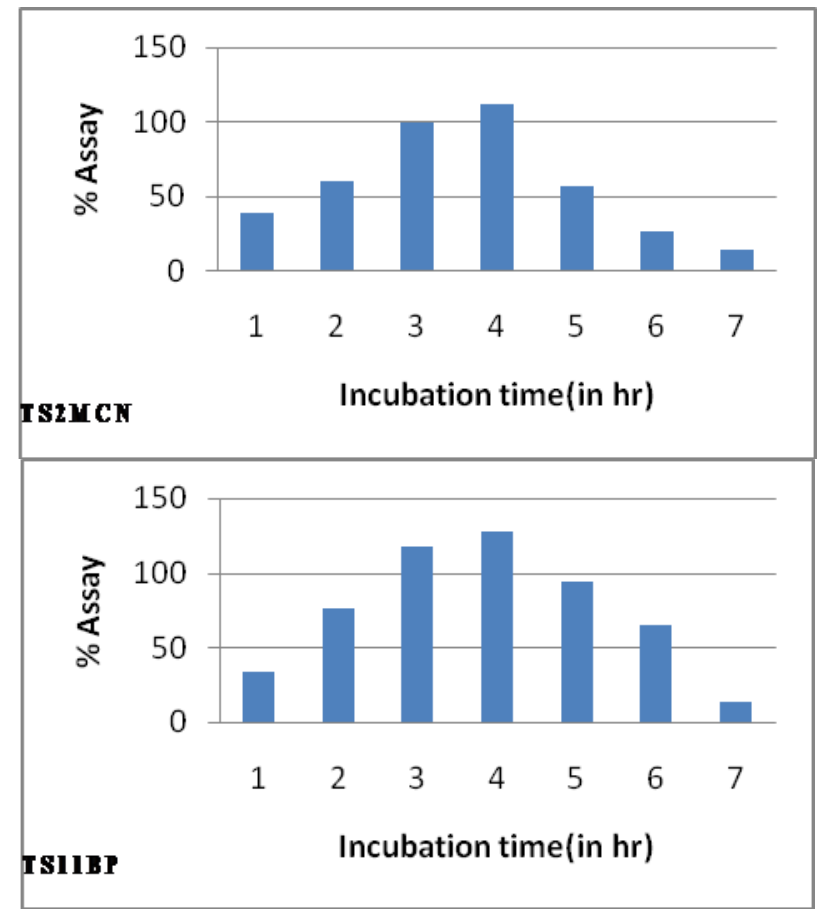

Figure 6: Effect of Incubation time on Lipase production by Two Strains
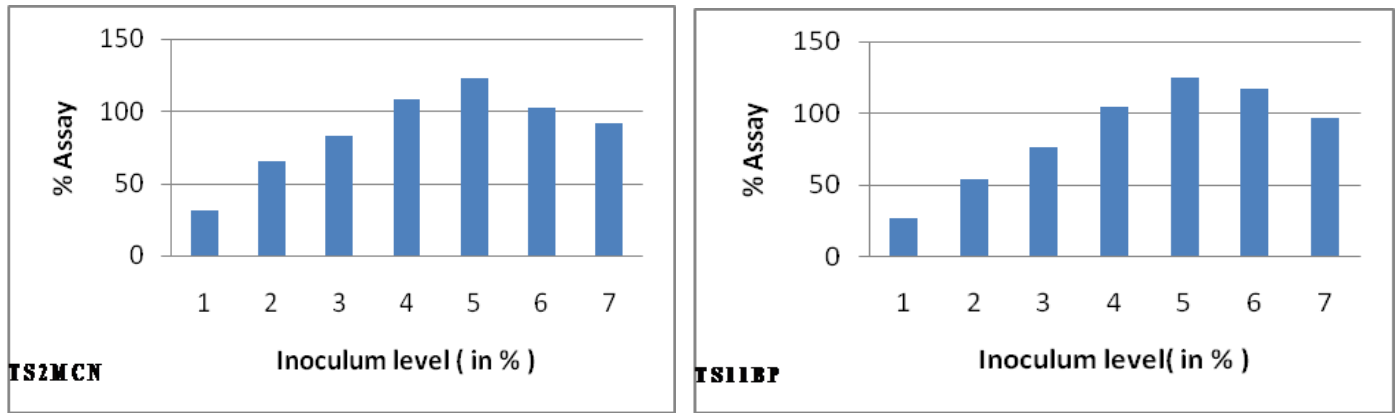

Figure 7: Effect of Inoculums level on Lipase production by Two Strains

\subsubsection{Effect of micro nutrients on lipase production:}

Micro nutrients include Vitamins and Amino acids, these two parameters were also termed as additional nourishing agents added to the medium for efficient growth of bacterial isolates. Different Vitamins such as Riboflavin, Ascorbic acid, Biotin, PyrodoxineHCl, Folic acid, Nicotinic acid and ThamineHCl were added to the medium in $0.01 \%$ concentration and assayed for selecting the optimum vitamin source. Amino acids such as L-Lysine, L-Luecine, L-Histidine, Tyrosine, Tryptophan, Aspargine, Glycine, D-Alanine, Cysteine, Arginine and Phenylalanine were added in the concentration of $0.01 \%$ and best amino acid giving maximal production of lipase was selectedthrough optimization studies by comparing assay values.

Table 10: Effect of Vitamins on bacterial isolates

\begin{tabular}{|c|c|c|c|c|c|}
\hline \multicolumn{2}{|c|}{ Bacterial isolate } & \multicolumn{2}{c|}{$\begin{array}{c}\text { Achromobacter xylosoxidans } \\
\text { (TS2MCN) }\end{array}$} & $\begin{array}{c}\text { Bacillus thuringiensis } \\
\text { (TS11BP) }\end{array}$ \\
\hline S.NO & Vitamins & IU units & $\%$ Assay & IU units & $\ldots .6$ \\
\hline 0 & Standard & 3.90 & $\ldots .$. & 1.60 & 35.00 \\
\hline 1 & Riboflavin & 2.50 & 64.10 & 0.56 & 124.37 \\
\hline 2 & Ascorbic acid & 3.99 & 102.30 & 1.99 & 65.62 \\
\hline 3 & Biotin & 3.01 & 77.17 & 2.22 & 138.75 \\
\hline 4 & PyrodoxineHCl & 4.88 & 125.12 & 1.88 & 117.50 \\
\hline 5 & Folic acid & 3.89 & 99.74 & 1.23 & 76.87 \\
\hline 6 & Nicotinic acid & 2.00 & 51.28 & 0.24 & 15.00 \\
\hline 7 & ThamineHCl & 1.55 & 39.74 & & \\
\hline
\end{tabular}


Table 11: Effect of Amino acids on bacterial isolates

\begin{tabular}{|c|c|c|c|c|c|}
\hline \multicolumn{2}{|c|}{ Bacterial isolate } & \multicolumn{2}{c|}{ Achromobacter xylosoxidans (TS2MCN) } & \multicolumn{2}{c|}{$\begin{array}{c}\text { Bacillus thuringiensis } \\
\text { (TS11BP) }\end{array}$} \\
\hline S.NO & Amino acids & IU units & \% Assay & IU units & $\ldots \ldots$. \\
\hline 0 & Standard & 3.90 & $\ldots \ldots \ldots$ & 1.60 & 124.37 \\
\hline 1 & L-Lysine & 3.99 & 102.30 & 1.99 & 30.62 \\
\hline 2 & L-Luecine & 4.88 & 125.26 & 0.49 & 96.87 \\
\hline 3 & L-Histidine & 2.88 & 73.84 & 1.55 & 156.25 \\
\hline 4 & Tyrosine & 4.66 & 119.48 & 2.50 & 144.37 \\
\hline 5 & Tryptophan & 4.51 & 115.64 & 2.31 & 33.12 \\
\hline 6 & Aspargine & 0.53 & 13.58 & 0.53 & 94.70 \\
\hline 7 & Glycine & 2.77 & 71.02 & 1.51 & 90.00 \\
\hline 8 & D-Alanine & 2.01 & 51.53 & 62.44 & 35.00 \\
\hline 10 & Cysteine & 2.44 & 14.35 & 0.56 & 14.37 \\
\hline 11 & Arginine & 0.56 & 39.74 & 0.23 & 34.37 \\
\hline
\end{tabular}

In our study it was depicted that medium supplemented to Achromobacter xylosoxidans (TS2MCN) with PyrodoxineHCl produced higher yield of lipase $4.88 \mathrm{IU} / \mathrm{ml} / \mathrm{min}$ compared to Bacillus thuringenesis (TS11BP) which yielded $2.22 \mathrm{IU} / \mathrm{ml} / \mathrm{min}$ of lipase. When compared to other vitamin sources like Ascorbic Acid, Folic Acid, Biotin and other vitamin sources Pyrodoxine $\mathrm{HCl}$ was best vitamin source for maximum lipase production. In case of Amino acids, Achromobacter xylosoxidans (TS2MCN) bacterial medium containing LLuecineproduced $4.88 \mathrm{IU} / \mathrm{ml} / \mathrm{min}$ of lipase which was comparatively high than Bacillus thuringenesis(TS11BP) yielding $2.50 \mathrm{IU} / \mathrm{ml} / \mathrm{min}$ with Tyrosine supplemented medium.
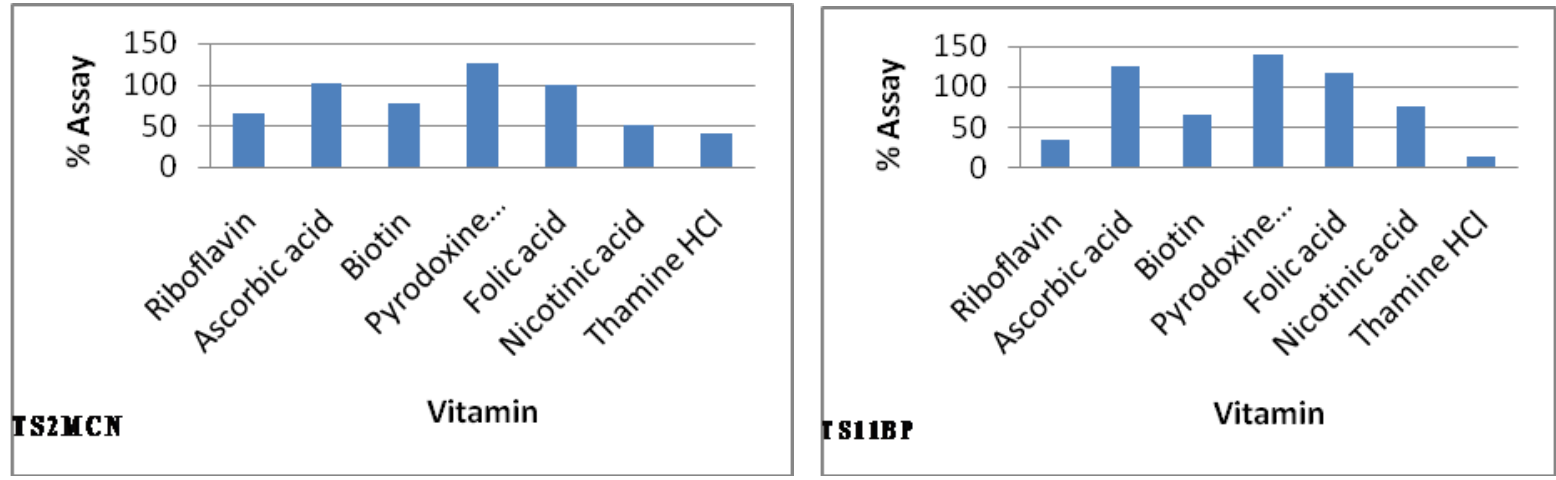

Figure 8: Effect of Vitamins on Lipase production by Two Strains
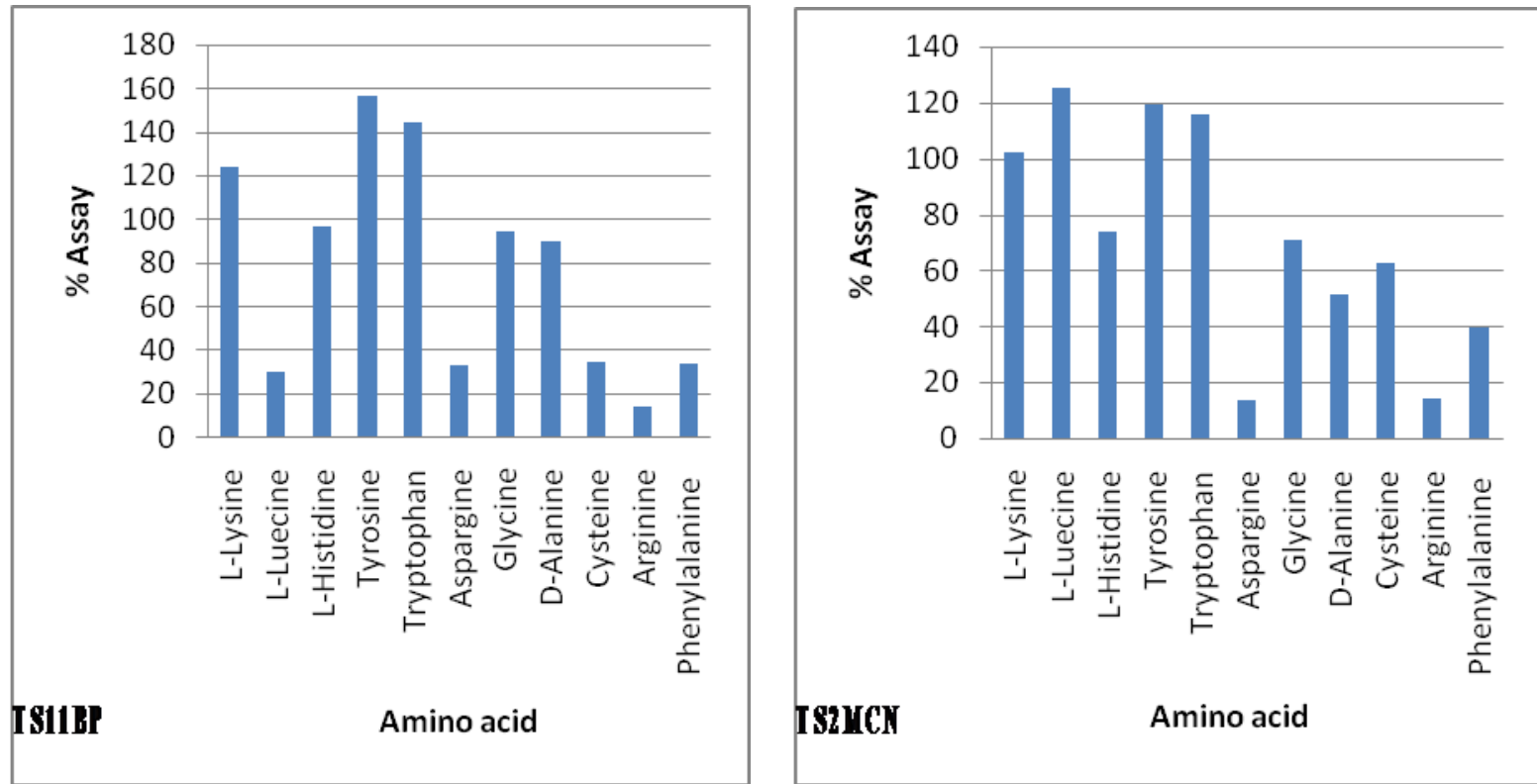

Figure 9: Effect of Amino acids on Lipase production by Two Strains 


\section{Conclusion:}

Laboratory scale optimization of some of fermentation conditions for lipase production by Bacterial isolates in submerged process was carried out. It can be concluded from the above studies, Achromobacter xylosoxidans (TS2MCN) and Bacillus thuringiensis (TS11BP) based on the high enzyme yields it could be considered as useful source of lipase enzyme production and has the potential for industrial applications. The optimum conditions of various growth parameters were stated by optimization studies. Lipases were in great demand these days owing to their numerous biotechnological applications. Further research on production, characterization and purification of lipases through optimization of process parameters such as $\mathrm{pH}$, temperature and various substrate utilizations would reveal those strains with higher lipase production potential.

\section{References:}

[1] Veeraragavan K. (1990) Anal Biochem.,186, 301-305.

[2] Thomsan C.A., Delaquis P.J., Mazza G. (1999) Crit. Rev. Food Sci. Nutr., 39, 165-187.

[3] Ashok P., Benjamin S., Soccol C.R., et al. (1999) Biotechnol Appl. Biochem., 29, 119-131.

[4] Falony G., Armas J.C., Mendoza J.C.D., Hernández J.L.M. (2006) Food Technol. Biotechnol., 44, $235-240$.

[5] Sharma R., Chisti Y., Banerjee U.C. (2001) Biotechnol. Adv., 19, 627-662.

[6] Gupta R., Gupta N., Rathi P. (2004) Appl. Microbiol. Biotech-nol., 64, 763-781.

[7] Priest F.G. (1992) Encyclopedia of Microbiology, Acadamic Press, New York, 2, 8193.

[8] E.A. Falch, Industrial enzymes- developments in production and application. Biotechnology Adv., 1991, 9: 643-658

[9] T. Fuji, T. Tatara and M. Minagawa, 1986. Studies on application of lipolytic enzyme indetergent industries. J. Am. Oil Chem. Soc., 1986,63: 796-799.

[10] E. Sirisha, N. Rajasekar and M. Lakshmi Narasu, Isolation and Optimization of Lipase Producing Bacteria from Oil Contaminated Soils. Advances in Biological Research, 2010, 4(5): 249-252, 2010.

[11] P. Bajpai, Application of enzymes in the pulp and paper industry. BiotechnolProgr, 1999, 15: 147-157

[12] K. Nakamura, and T. Nasu, Enzyme Containing Bleaching Composition, Japanese Patent, 1990, 2: $208-400$.

[13] K.E. Jaeger, S. Ransac, B.W. Dijkstra, C. Colson, M. Heuvelvan, O. Misset, FEMS. Microbiol. Rev., 1994, 15, 29- 63.

[14] F. Hasan, A.A. Shah, A. Hameed, Enzyme Microb. Technol., 2006, 39, 235-251.

[15] J. Vakhlu, A. Kour, Electr. J. Biotechnol., 2006, 9, 69-85.

[16] Y. Wang, K.C. Srivastava G.J. Shen, H.J. Wang, J. Ferment. Bioeng., 1995, 79: 433- 438.

[17] K.E. Jaeger, T .Eggert, Curr. Opinion Biotechnol.,1998, 13, 390-397.

[18] U.T. Bornscheuer, C. Bessler, R. Srinivas, S.H. Krishna, Trends Biotechnol., 2002,20, 433-437.

[19] Buchanan RE, Gibbons NE. [1974] Bergey's of determinative bacteriology. America: United States of America 529-563.

[20] Mohammad Badrud Duza and S A Mastan, "Isolation, Characterization and Screening of Enzyme Producing bacteria from different soil samples" Int J Pharm Bio Sci 2013 July; 4(3): (b) 813 - 824

[21] V.K. Winkler, M. Stuckmann, J. Bacteriol.,1979, 138, 663-670. 